\title{
The conception of Humanoid body fluids
}

\begin{abstract}
Advantaged with our organically flexible body and internal circulatory system, we humans could stay balanced even in rapid and difficult movements and under extreme conditions in vibrating and rugged environment. However, stability of locomotion has become a challenge for humanoids that are constructed with rigid body parts connected by joints. The awareness of this contrast could shed light on how to ease the task of balancing the locomotion of humanoids by making use of body fluids inside their bodies. With the help of some simple schematics, this short article would offer a brief conceptual (metaphysical) discussion on the benefit of introducing body fluids to the construction of humanoids.
\end{abstract}

Volume 6 Issue 2 - 2020

\author{
Rongqing Dai \\ Freelance philosopher, New Jersey, USA
}

Correspondence: Rongqing Dai, Ph. D., freelance philosopher, New Jersey, USA, Tel 00I-732-395-3163,

Email ronald_dai@yahoo.com

Received: May 07, 2020 | Published: June 17, 2020

Keywords: humanoid, body fluids, mobility, rigid, bipedal locomotion

\section{Introduction}

Since ancient times, creating automated machines, looking and acting like humans or animals, has been a dream of the mankind. While the human-looking robots bleeding blue blood when getting injured still exist only on screen, the need of body fluids for humanoids (i.e. robots resembling the shape of human body) could become real because of the difficult task of making a bipedal machine to walk with ease as humans, not for the circulation of biological cells or nutrients, but for helping the robot to stay balanced when accomplishing difficult movements in complicated environments.

Having the robots to resemble humans might offer many conveniences for the interest of robotics and AI scientists, ${ }^{1,2}$ and tens of thousands of papers have been published during the past decade for the subject of how to pass through the so-called uncanny valley or related subjects, in a collective effort to make robots to be accepted into human society as assistants to humans, and eventually as normal members like us.

Nonetheless, while standing and walking may come easy for humans, the implementation of a machine that could stand and walk with two legs in variegated postures to fit in realistic life circumstances is a very difficult task. So far humanoids have been basically constructed with rigid body parts connected by joints (with or without artificial skin), and accordingly their movements are modeled by the mechanics of the system of solid objects with required degrees of freedom. This would be inevitably far from the mechanics of real humans with flexible body parts in real life situations, and one of the important reasons would be because with rigid body parts it is very difficult to achieve the stability brought by the flexibility of the deformable mass, despite some studies have claimed a satisfactory match between the rigid model and real humans for limited scenarios (e.g. Hoang et al 2018 ${ }^{3}$ ).

Therefore, we might help to ease the task of keeping bipedal locomotion of humanoids in balance by introducing a system (or systems) of body fluids into their inner structures. Besides, by using body fluids, we might also have some other conveniences which sometimes could be difficult to achieve for products with rigid body parts only.

\section{Benefits of body fluids for humanoids}

Obviously, the balance of a humanoid at all velocities is not something that can be easily handled in a similar way to self- balancing board ${ }^{4,5}$ or other like instruments that the public has become familiar with for the past couple decades. With a humanoid of many rigid body parts in various postures, we would face not only the issue of managing the center of mass during complicated movements at varying accelerations as a whole with all available degrees of freedom (and thus in all possible directions), but also the issue of balancing the dynamic torques at all joints with all the local angular momentums and the local gravitational forces.

As a matter of fact, when positioning or moving our bodies in the varieties of complicacy in real life events, the very nuances in the displacement of mass center could cause imbalance of the body and lead to stumble. This is especially true in some highly challenging performances, when balancing is an issue of life and death, such as high wire walking, acrobatics, gymnastics, big wave surfing, and short track speed skating or figure skating, or even in some slow and peaceful motions like tai chi when the smooth movement of the holistic inner world of the body is the key to the high quality of training. Due to the universal effect of gravity, compared to the humanoids with rigid body parts, the existence of our circulatory system of body fluids, in addition to our deformable organic body parts, would undoubtedly help to achieve the effect of sensitive balance in the abovementioned circumstances, when a subtle displacement of the center of the total weight could make a big difference in the result. Particularly, when the subject comes to a slanting posture (which could often be required for the program of performance) after a sudden stop of movement, the chance of tipping over could be reduced by intentionally moving the center of mass backward in advance or in the moment, which is hard to achieve by adjusting the positions of rigid body parts without internal movements.

Therefore, a straightforward logic would tell us that adding a system of body fluids could help to reduce the difficulty of managing the balance of a humanoid with its standing and walking in various postures. Besides, once the concept of body fluids of humanoids comes into life, we might also have other mechanical advantages brought by the use of the body fluids inside humanoids.

\section{The conception of the potential implementation}

Conceptually, the system of body fluids for mechanical benefits could be implemented through different approaches, either highly sophisticated bionic systems with a heart-like central pump cooperating with distributed local microscopic regulators, or a simple computer controlled distributed system of tubes and pistons for moving body 
fluids locally and between different parts of the body. Figure 1 is a schematic drawing that conceptually shows a simple tube and piston unit which could be used to move body fluids either driven by a local motor or pushed by body parts when the position of body changes. Figure 2 is a schematic drawing that conceptually shows how body fluids might be moved around between different parts of the body.

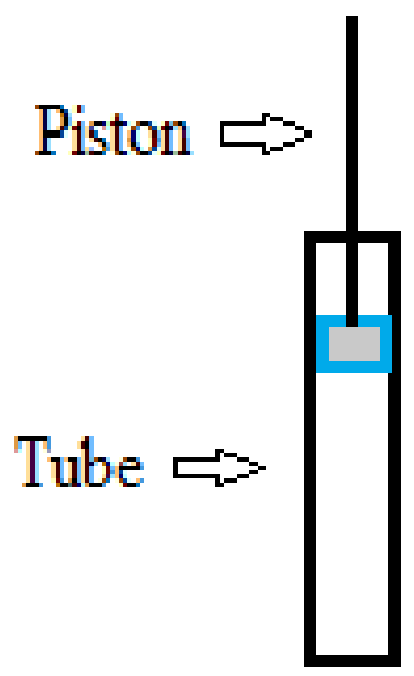

Figure I Schematic of simple tube-piston unit.

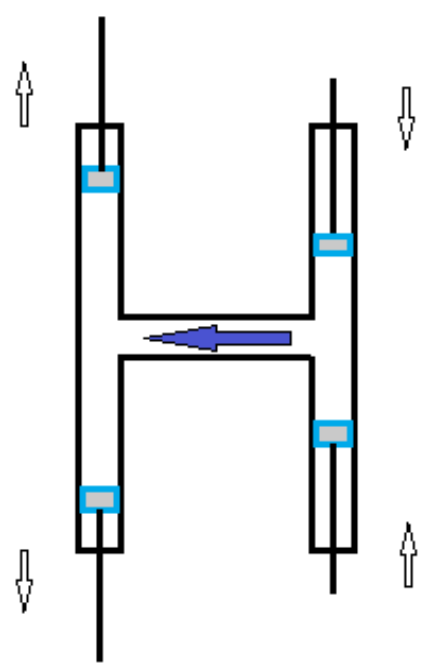

Figure $\mathbf{2}$ Schematic of fluid movement in the tube-piston system.

In general, no matter for shifting the center of mass or for the sake of other benefits that body fluids could bring about, the local regulators (valves or pistons, etc) for directing the movement of body fluids should be controlled by the central logic processor(s); but on the other hand, local automation might not always need to be digitally programmed, and sometimes we might make use of the local mechanical convenience itself. For example, as conceptually shown in Figure 3, we might add a mechanically deformable compartment at a joint of leg, and fill it with fluids. With a partition (as illustrated by the red line segment) within the compartment, when the leg is bent, the volume of the front partition could be enlarged and the volume of the rear partition could be compressed, and thus the fluid pressure in the rear partition would increase to create a counter torque against the bending so that the leg would not be overly bent by gravity. Of course, we could also add an inducing line to the rear partition to let out the excessive fluid when a complete bending is wanted.

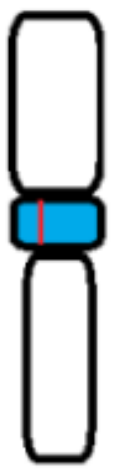

(a)

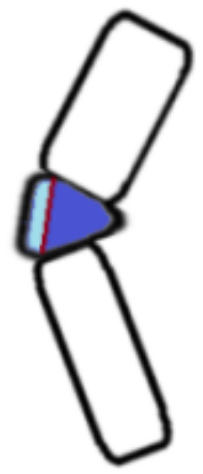

(b)
Figure 3 Schematic of the increased local fluid pressure to balance the local torque of bending. (a) without bending; (b) with bending.

\section{The variety and pressure of body fluids}

We might use different fluids for different types of functionalities within a humanoid body. The fluid for creating local compressive pressure might not be the same as the fluid for shifting the center of mass. Even for the same type of functionality at different parts of the body, it is not necessary to have the same unique fluid. We might use heavier fluids (e.g. mercury) when the local space is limited, and lighter fluids for more spacious places.

Besides, in order to assure that the humanoid could shift its center of mass more smoothly at will when walking on rugged terrain or doing physically complicated maneuvers, we need to have a high responsiveness of the whole system of fluids, and this would require high values of bulk modulus of all the fluids inside the humanoid, which could often mean high values of fluid pressure. Accordingly, we would need good quality of pressure sealing for the tubes and connections of the inner body fluid system.

\section{Final remarks}

The mobility of body fluids could conceptually help to ease the difficult task of bipedal locomotion of a humanoid with cumbersome rigid body parts (connected by multiple joints), through the continuous shift of the center of mass, when making complicated physical maneuvers. But it would take engineering ingenuity to practically implement the conception of humanoid body fluid system as discussed in this writing, which would undoubtedly involve complicated calculation and simulation. Nonetheless, in order to make future humanoids truly act like humans, the introduction of body fluids would be an indispensable step.

\section{Funding}

None.

\section{Acknowledgments}

None.

\section{Conflicts of interest}

The author declares that there was no conflict of interest. 


\section{References}

1. Single Gadget. The Future of Humanoid Autonomous Robots. 2016.

2. Brandon A. When Robots Are Indistinguishable From Humans, What Will Be Inside Them? 2017.

3. Hoang KL, Wolf S, Katja M. Benchmarking Stability of Bipedal Locomotion Based on Individual Full Body Dynamics and Foot Placement Strategies-Application to Impaired and Unimpaired Walking. Front. Robot. AI. 2018.
4. How Do Hoverboards Balance?

5. Wikipedia. Self-balancing scooter. 2020. 